\title{
National Institutes of Health Center for Regenerative Medicine: Putting Science into Practice
}

Mahendra Rao*

\section{ABSTRACT}

The field of regenerative medicine has been revolutionized by breakthroughs in stem cell biology, gene engineering, and whole-genome sequencing. These advances are not only scientific or medical but have also advanced how we conceptualize regenerative medicine. The progenitive research that proceeded as well as a substantial part of the funding that supported these discoveries were provided by the National Institutes of Health $(\mathrm{NIH})$. Now, perhaps more than ever, the $\mathrm{NIH}$ has a vital role to play in the translation of science into clinical practice. The $\mathrm{NIH}$ is uniquely positioned to coordinate interactions between the different institutes and other arms of the government, as well as international organizations. Efforts of researchers in the United States both within and without the NIH are supported by a number of mechanisms, including specialized workshops, and the support of developing small-scale industry. Additionally, the NIH has stepped up to provide necessary infrastructure in areas of regenerative medicine where the medical need might be apparent but might be currently infeasible or unattractive to private-sector investment. This article will discuss these perhaps lesser-known activities of the $\mathrm{NIH}$, which I believe have continued and will continue to contribute to the role of stem cell research in translating science into regenerative medicine.

\section{The National Institutes of}

\section{Health and its role in}

\section{putting medical science into practice}

The National Institutes of Health (NIH) plays a pivotal role in funding medical

NIH Center for Regenerative Medicine, Bethesda, Maryland.

*(Correspondence: mahendra.rao@nih.gov) research and leads the efforts at translating that science into clinical practice in the United States. The dollar amount invested specifically into stem cell research approximates a billion dollars a year (www.nih.gov). However, NIH support of regenerative medicine is not limited simply providing research dollars. To enable the translational efforts in the United States, the NIH needs to not only anticipate how scientific breakthroughs can inform regenerative medicine but also proactively lead reconceptualization of how therapeutic development can be supported and implemented.

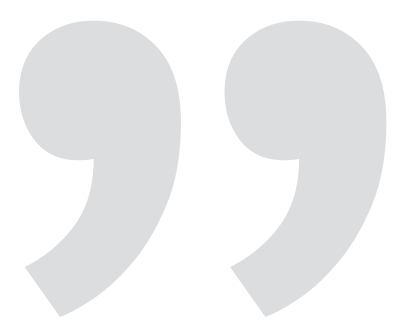

The dollar amount invested specifically into stem cell research by the $\mathrm{NIH}$ approximates a billion dollars a year.

\section{Proactive leadership rather than reactive stewardship}

The NIH is the premier source of medical funding in the United States and, as such, its allocation efforts strongly influence current and future strategies of development in regenerative medicine. I contend that the NIH has and will continue to anticipate the dizzying pace with which research discoveries are being advocated for and indeed, in some cases, 
translated to clinical practice. From scientific, medical, and regulatory standpoints, the NIH must take an anticipatory leadership role rather than play catch-up to a field that requires proactive, not reactive, stewardship [1].

It appears to the author that the field has accelerated by taking advantage of discoveries made in other fields and applying these breakthroughs to regenerative medicine. For example, the Nobel Prizewinning works on pluripotency of Dr. Gurdon and Dr. Yamanaka, [2,3] synergized with that of Dr. Mario Capecchi [4,5], develop homologous recombination in human stem cells. Such techniques to redefine not only the potentiality but also the genetic complement of cells appear powerful and useful. However, their safe implementation even as a screening tool requires characterization and standardization. This in turn is informed by the now available ability to test, confirm, and retest cells in culture conditions whose properties we understand in detail thanks in turn to the advances in next-gen sequencing [6-8].

Next-gen sequencing technology is now available not only for therapeutic screening development but also in the diagnostic arena. Current and future applications include testing for possible heritable conditions as well as identifying genetic mutations or modifiers in patients displaying symptoms that defy definitive diagnosis. The neurological condition suffered by the Beery twins would likely remain undiagnosed were it not for the successful diagnosis based on the DNA sequencing and subsequent treatment [101]. This example illustrates the power of these techniques. We now have the ability, but also the responsibility, to determine how they are applied $[9,10]$.

The general public's concept of what role stem cells would play in medicine still likely focuses on their possible direct therapeutic application, whether it is cord blood storage services, mesenchymal-derived tissue repair, or the more publicized clinical trials investigating neurological repair using neural or embryonic stem

\section{The NIH Center for Regenerative Medicine}

The NIH Center for Regenerative Medicine aims to provide the infrastructure to support and accelerate the clinical translation of stem cell-based technologies and to develop widely available resources to be used as standards in stem cell research.

For a variety of patient populations, the center facilitates generation of induced pluripotent stem cells, as well as the derivation or isolation of other types of stem cells. The center makes available a range of adult stem and progenitor cell populations, as well as the protocols and standard operating procedures used for their derivation, culture, and differentiation. The center collaborates in the United States and internationally-with governments, research institutions, and commercial entities-to ensure that efforts are coordinated and standard policies are in place to minimize roadblocks to advancing stem cell technologies. The Center for Regenerative Medicine is funded by the NIH Common Fund.

More information can be found at: www.crm.nih.gov

cell-based populations. Perhaps less dramatic, but possibly of more immediate impact, has been seen in the realms of therapeutic screening and toxicological testing.

Cardiomyocytes derived from human pluripotent cells are now available on a large scale from a number of companies and have been adopted as the gold-standard test in early-stage drug screening $[10,11]$. Given the cost of drug development, the ability to screen drugs for any condition against possible cardiac toxicity is a clear example of the efficacy and cost-effectiveness of such developments [12]. The difficulties in deriving homogeneous populations from pluripotent stem cells are acknowledged, but solvable. The relevance of developing better, more reliable human-based in vitro screening tools will continue to attract both public and private investment in all spheres of medical treatment whether it is for widespread conditions such as diabetes or for orphan and rare disorders. The NIH allocates resources and identifies experts and sets up think tanks to receive feedback on how best to move forward [13].

To obtain the best return on research dollars, the NIH, along with its direct research support, has partnered with other government agencies such as the Defense Advanced Research Projects Agency, the
Department of Defense, the Veterans Administration, as well as with the National Institute of Science and Technology and the Environmental Protection Agency to help coordinate funding efforts and has begun to develop shared databanks. The NIH worked to help coordinate such activity and develop public-private partnerships [14]. An important component of public-private partnership that has been the cornerstone of innovation in biotechnology are the Small Business Innovation Research (SBIR), Small Business Technology Transfer (STTR), and advanced technology funding programs. The NIH has encouraged stem cell companies to register and apply for these grants and has worked at the institute level to ensure that companies could access the requisite technology to be competitive in the bidding process [102].

The NIH realized that current funding programs may not be suited for translational research and that necessary infrastructure would need to be developed to allow for successful translation. As the $\mathrm{NIH}$ engaged in discussion with stakeholders, they identified a need to introduce larger, longer-term grants to enable preclinical studies to be successfully completed by academic investigators [13].

Three major initiatives by Dr. Collins deserve special mention: the National Cen- 
ter for Advancing Translational Science was established with the task of accelerating translational science, including work with stem cells, in screening and toxicology as well as to help bridge the valley of death that exists because of a dearth of funding from traditional investment sources (www.ncats.nih.gov). The $\mathrm{NIH}$ has identified the Clinical Center (http://clinicalcenter.nih.gov) as an invaluable resource and developed plans to allow access to NIH-funded investigators worldwide. Dr. Gallin, the clinical center chief, has identified different ways to work with the center [103], including work with stem cells. More recently, Dr. Collins established the NIH Center for Regenerative Medicine (NIHCRM) to help identify the roadblocks that exist for this field and to develop creative solutions to resolve such roadblocks (www.crm.nih.gov).

Regulatory issues have been identified as a major impediment to translational advancement. To encourage and support investigators, the NIH has developed model consent forms, Material Transfer Agreements (MTAs), and identified patent issues and negotiated appropriate limited-use label licenses to enable access to appropriate service providers. The NIHCRM has further generated induced pluripotent stem cell lines, including engineered reporter lines, and deposited them for distribution worldwide with minimal restrictions and at a reasonable cost (see, e.g., [104]). The NIH has also developed Production Assistance for Cellular Therapies (PACT) centers for manufacturing clinical-grade cells, and university-based screening centers to allow investigators access to resources that would be difficult to generate for any one individual group. The NIH has also developed and centralized standard operating procedures and reagents so that such technology can be widely accessed and replicated. This promotes a shared understanding of what a particular methodology entails, but just as importantly a shared agreement of how results can be appropriately interpreted from different sites.

Although the NIH believes that its efforts discussed above will play an important role in accelerating the development of cell-based therapeutic intervention, it recognizes that many of the old models of drug delivery may not work and new business models will have to be supported. Examining the field, one can broadly divide new efforts into three different groups. Gene therapy companies have begun to adopt advances in vector design, self-inactivating vectors, or integration-free gene delivery technologies and have initiated clinical trials. Three different approaches are being adopted: use of cells as delivery vehicles in which the product is inserted into a safe harbor site using vectors that allow conditional delivery that is not epigenomically silenced for the lifetime of the individual; a knockout strategy in which the abnormal product is deleted using precision excision methods; and a repair strategy in which the specific defect is corrected. Individual groups and companies are exploring these technologies for therapy in diseases such as cancer, AIDS, and lysosomal storage disorders $[15,16]$.

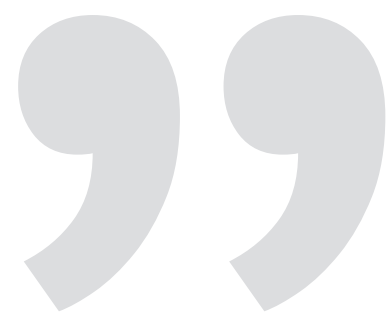

\section{Gene engineering breakthroughs coupled with the ability to make differentiated cells of various kinds will allow for functional cures.}

Several hospital- and institute-based services have begun to adopt biomaterial engineering services and combine them with cell culture techniques to build 3D structures. These include structures such as the trachea, esophagus, valves, bladder, and sheets of cells. Companies that provide specialized bioreactors to enable this sort of growth have emerged, and novel methodologies to image the growing tissue have been developed [17-19].

It is hard to imagine fitting these efforts into the centralized manufacture and global distribution model that traditional BioPharma companies have used. New models, however, mean new infrastructure, with an evolved understanding of party involvement and how the stakes, the risks, and the returns are distributed among those parties [20-23]. Time will tell us what will work best, but "best is the enemy of good," and the time to start proactively attempting to develop a solution is now.

It seems to this writer that the gene engineering breakthroughs coupled with the ability to make differentiated cells of various kinds will allow for functional cures. The marrying of biomaterial science with stem cell technology is limited now only by our inability to construct the necessary vasculature to allow nutrient delivery in synthetic constructs that are more than 8 or 10 cell layers thick. However, the developing ability to print organs layer by layer, and the isolation of endothelial cells in large numbers suggest that we are close.

The NIH has recognized that it needs to constantly evaluate its priorities and that allocations must remain responsive, but at the same time recognizes that individual efforts at translational development require sustained investment to allow clinical evaluation and eventual successful intervention. The NIH will continue its efforts to gather information and obtain feedback and encourages readers to write or respond to the request for information that the NIH publishes.

The NIH believes that its role in promoting regenerative medicine includes not just funding but also proactively stewarding the field through anticipating the shifting needs and shortcomings as well as likely developments. This will be achieved by continuous dialog and consultation to take a lead in not only scientific development but also standardized testing and reagents, 
regulatory clarity, and clinical trial implementation. The NIH has a unique role to play in the support and encouragement of the coordination of public and private efforts in translational research toward improved health goals. These strategies will result in the safe, more cost-effective translation of medical research efforts into clinical practice.

\section{Disclaimer}

The opinions expressed in this article reflect my personal views and do not in any way represent or are indicative of the views of my employer.

\section{Author disclosure statement}

No competing financial interests exist.

\section{REFERENCES}

\section{Primary Literature}

1. Rao MS and FS Collins. (2012). Steering a new course for stem cell research: NIH's intramural Center for Regenerative Medicine. Stem Cells Transl Med 1:15-17. 2. Gurdon JB and JA Byrne. (2003). The first half-century of nuclear transplantation. Proc Natl Acad Sci USA 100:8048. 3. Takahashi K, K Tanabe, M Ohnuki, M Narita, T Ichisaka, K Tomoda and S Yamanaka. (2007). Induction of pluripotent stem cells from adult human fibroblasts by defined factors. Cell 131:861-872.

4. Thomas KR and MR Capecchi. (1987). Site-directed mutagenesis by gene targeting in mouse embryo-derived stem cells. Cell 51:503-512.

5. Capecchi MR. (1989). Altering the genome by homologous recombination. Science 244:1288-1292.

6. Ginsburg GS and HF Willard. (2009). Genomic and personalized medicine: foundations and applications. Transl Res 154:277-287.

7. Korf BR and HL Rehm. (2013). New approaches to molecular diagnosis. JAMA 309:1511-1521.
8. Rao AV and BD Smith. (2013). Are results of targeted gene sequencing ready to be used for clinical decision making for patients with acute myelogenous leukemia? Curr Hematol Malig Rep 8:149155.

9. Juengst ET, RA Settersten Jr, JR Fishman and ML McGowan. (2012). After the revolution? Ethical and social challenges in "personalized genomic medicine." Per Med 9:429-439.

10. Himmel HM. (2013). Drug-induced functional cardiotoxicity screening in stem cell-derived human and mouse cardiomyocytes: effects of reference compounds. J Pharmacol Toxicol Methods 68:97-111.

11. Knollmann BC. (2013). Induced pluripotent stem cell-derived cardiomyocytes: boutique science or valuable arrhythmia model? Circ Res 112:969-976; discussion 976.

12. Liang P, F Lan, AS Lee, T Gong, V Sanchez-Freire, Y Wang, S Diecke, K Sallam, JW Knowles, et al. (2013). Drug screening using a library of human induced pluripotent stem cell-derived cardiomyocytes reveals disease-specific patterns of cardiotoxicity. Circulation 127:1677-1691.

13. Williams RL, SB Johnson, SM Greene, EB Larson, LA Green, A Morris, D Confer, G Reaman, R Madigan and J Kahn; Principal Investigators of Clinical Research Networks Initiative. (2008). Signposts along the NIH roadmap for reengineering clinical research: lessons from the Clinical Research Networks initiative. Arch Intern Med 168:1919-1925.

14. Rao M. (2013). Public private partnerships: a marriage of necessity. Cell Stem Cell 12:149-151.

15. Garate Z, BR Davis, O QuintanaBustamante and JC Segovia. (2013). New frontier in regenerative medicine: sitespecific gene correction in patient-specific induced pluripotent stem cells. Hum Gene Ther 24:571-583.

16. Keung EZ, PJ Nelson and C Conrad. (2013). Concise review: genetically engineered stem cell therapy targeting angiogenesis and tumor stroma in gastrointestinal malignancy. Stem Cells 31:227-235.
17. Drewa T, J Adamowicz and A Sharma. (2012). Tissue engineering for the oncologic urinary bladder. Nat Rev Urol 9:561-572.

18. Vrana NE, P Lavalle, MR Dokmeci, F Dehghani, AM Ghaemmaghami and A Khademhosseini. (2013). Engineering functional epithelium for regenerative medicine and in vitro organ models: a review. Tissue Eng Part B Rev. [Epub ahead of print]; DOI:10.1089.ten.teb. 2012.0603.

19. Payne KF, I Balasundaram, S Deb, L Di Silvio and KF Fan. (2013). Tissue engineering technology and its possible applications in oral and maxillofacial surgery. Br J Oral Maxillofac Surg. [Epub ahead of print]; DOI:10.1016/j. bjoms.2013.03.005.

20. Kawamoto K, DF Lobach, HF Willard and GS Ginsburg. (2009). A national clinical decision support infrastructure to enable the widespread and consistent practice of genomic and personalized medicine. BMC Med Inform Decis Mak 9:17.

21. Gaspar R, B Aksu, A Cuine, M Danhof, MJ Takac, HH Linden, A Link, EM Muchitsch, CG Wilson, P Ohrngren and L Dencker. (2012). Towards a European strategy for medicines research (20142020): The EUFEPS position paper on Horizon 2020. Eur J Pharm Sci 47:979-987. 22. Fackler JL and AL McGuire. (2009). Paving the way to personalized genomic medicine: steps to successful implementation. Curr Pharmacogenomics Person Med 7:125.

23. Abrahams E, GS Ginsburg and M Silver. (2005). The personalized medicine coalition: goals and strategies. Am J Pharmacogenomics 5:345-355.

\section{Websites}

101. http:/dystonia.thebeerys.com/Video/ TabId/89/VideoId/7/DNA-Sequencing 39s-Promise-For-Personalized-Medicine .aspx

102. http://oamp.od.nih.gov/contracts/ contract.htm

103. http://clinicalcenter.nih.gov/about/ news/newsletter.html

104. http://scrc.rutgers.edu/or www.wicell .org/home 